\title{
CONTINUATION OF CODIMENSION-2 EQUILIBRIUM BIFURCATIONS IN CONTENT
}

\author{
W. GOVAERTS*, YU. A. KUZNETSOV', AND B. SIJNAVE*
}

1. Introduction. We consider a dynamical system depending on parameters,

$$
\dot{u}=F(u, \alpha), \quad F: \mathbb{R}^{n} \times \mathbb{R}^{m} \rightarrow \mathbb{R}^{n},
$$

and its numerical study using CONTENT, an interactive software environment developed by Yu. A. Kuznetsov and V. V. Levitin, available together with a tutorial by anonymous ftp from ftp. cwi.nl in the directory /pub/CONTENT.

CONTENT can detect, compute and continue codimension-1 bifurcations of the solutions to the equilibrium equations

$$
F(u, \alpha)=0
$$

associated with (1.1). Limit points and Hopf points are computed using the methods developed in [21], [19], [12], [14], [13], [15], [20], [9], [1], [4], [23] and [11].

We discuss recent extensions to CONTENT to compute and continue all codimension-2 bifurcations of the solutions to (1.2). These are based on numerical methods developed in [9], [6], [7]; we restrict attention to the implementation of the methods in CONTENT. We deal with Bogdanov-Takens $(\mathrm{BT})$, zero-Hopf $(\mathrm{ZH})$, double Hopf $(\mathrm{DH})$, cusp $(\mathrm{CP})$ and generalized Hopf (GH) points. For the dynamical significance of these points we refer to [10] and [16]. We also explain how to detect codimension-3 equilibrium bifurcations associated with the degeneration of the linear terms of (1.1).

We discuss the structure of CONTENT, the basic ideas of the numerical methods, the way they are implemented, and give several examples.

Mathematical issues, such as manifolds of systems with a given bifurcation type, regularity of the linear systems, detection of codimension-3 singularities due to nonlinear terms of (1.1), relations to the universality of the unfolding in the natural parameters etc. will be published elsewhere.

2. Basic structure of the computational methods. We call each bifurcation type an object. We deal with the five codimension-2 objects (BT, $\mathrm{ZH}, \mathrm{DH}, \mathrm{CP}$, and $\mathrm{GH}$ ) and their interactions with the codimension-1

- Department of Applied Mathematics and Computer Science, University of Gent Krijgslaan 281 (S9), B-9000 Gent, Belgium. W. Govaerts is a research director of the Fund for Scientific Research FWO, Belgium.

tCentrum voor Wiskunde en Informatica, Kruislaan 413, 1098 SJ Amsterdam, The Netherlands and Institute of Mathematical Problems of Biology, Russian Academy of Sciences, Pushchino, Moscow Region, 142292 Russia. 


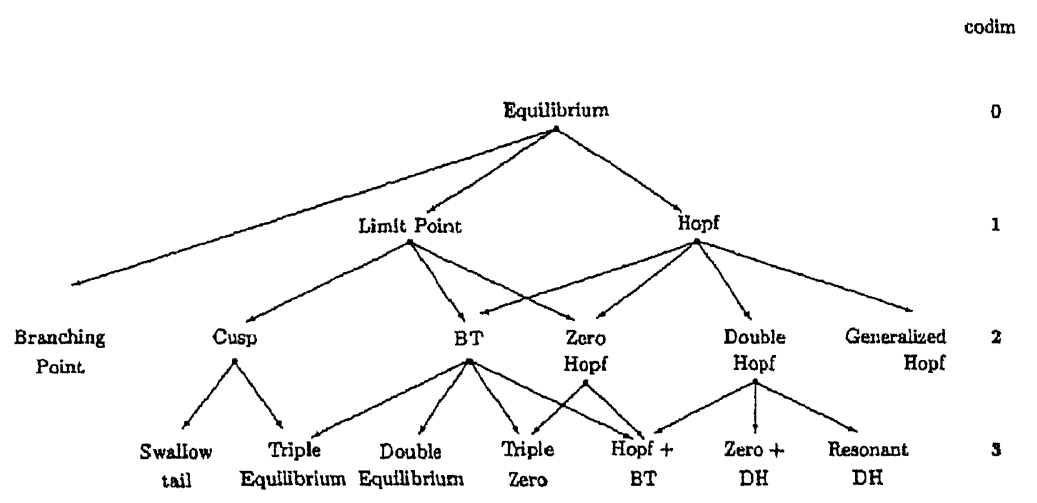

FIG. 1. Interactions between objects of codimension $0,1,2,3$.

objects (limit point and Hopf) as well as some codimension-3 objects (triple zero eigenvalue, resonant double Hopf, etc.). For an overview of the possible interactions, we refer to figure 1. There are several ways to compute and continue a given object. We call these computational schemes. To store a computed representative of any given object, CONTENT uses bifurcation data, namely, the equilibrium coordinates and critical parameter values (i.e., the values $u, \alpha$ from (1.1)) and certain other data that depend on the object but not on the computational scheme. This allows to compute an object using one computational scheme and switch to another scheme whenever necessary.

The kernel of CONTENT describes how objects are read from file, continued, detected and written to file. The user chooses the computational scheme and many details of the computation by using specially designed windows. The same continuation code is used in all computational schemes.

The variables of the continuation in a particular computational scheme are called the continuation data. They always include the equilibrium coordinates but not necessarily the other bifurcation data of the object. On the other hand, the continuation data may include variables not in the bifurcation data of the object. Apart from this, a computational scheme often uses variables which are not part of the continuation data but have to be initialized at the starting point and updated along the computed curve. These we call auxiliary data.

Suppose that a computational scheme $\mathcal{C}\left(O_{b}\right)$ has to be written for the object $O_{b}$. Logically, the first task is to write Starters for $\mathcal{C}\left(O_{b}\right)$. One needs as many Starters as source objects, i.e., objects from which $O_{b}$ can be continued. This includes $O_{b}$ itself but typically there are more. For example, a Hopf curve may start from a Hopf point (Hopf is an object) but also from a BT-point (a different object) or from a ZH-point (still another 
object). The Starters must read the bifurcation data of the source object, compute the bifurcation and continuation data for $\mathcal{C}\left(O_{b}\right)$ and initialize the auxiliary data.

Next, $\mathcal{C}\left(O_{b}\right)$ is mainly determined by its so-called Defining Equations. This is a system of equations in which the variables are the continuation data. The included parameters are called free parameters. The user decides at runtime which parameters are free in a given application but the number of free parameters is fixed in advance by the choice of the object: the number of free parameters has to be one more than the codimension of the object since continuation has to be possible. In particular, for all codimension- 2 objects three parameters must be free.

The continuation data usually contain more than just the equilibrium coordinates. For example, in a Hopf case they might include the imaginary part of the Hopf eigenvalue and, maybe, the components of the eigenvectors. But the number of defining equations has to be one less than the number of continuation data, again to allow continuation.

CONTENT needs the derivatives of $F$ but can compute these symbolically if desired. In fact, the user has three choices: to compute derivatives symbolically, by means of finite differences or by a user-supplied routine. Since bifurcations are typically defined by the derivatives of $F$, CONTENT usually also needs higher order derivatives of $F$; the user has the same choices.

In most cases it is necessary to refresh the auxiliary data of the computational scheme from time to time; their choice is indeed based on local information and optimal only in a part of the $(u, \alpha)$-space. Therefore one has to write an Adapter, i.e., a routine that updates the auxiliary data (the user decides the frequency of this updating; default, updating is done after every continuation step).

While computing a curve of $O_{b}$-points CONTENT is likely to encounter certain codimension- 3 bifurcation points. To investigate this, a number of test functions can be computed along the curve whose sign changes indicate that "something was encountered." Conceptually the simplest situation is that there is a one-to-one relation between the test functions and the potential bifurcations with higher codimension. In reality this is not always possible and sometimes one has to compare several test functions to decide what bifurcation point was found. But the starting point of a detection is always the sign change of a test function and the exact location of the critical point is found by a bisection method that computes the zero point of the test function. After that, a comparison with the values of other test functions leads to a decision. Writing a comparison table is of course part of the implementation work for $\mathrm{O}_{b}$.

Finally, the code should be able to stop at any user-chosen point and write to file the coordinates of the point and the bifurcation data of the object. 
3. Bogdanov-Takens. The chosen bifurcation data for BT-points consist only of the state and parameter values; the computational scheme uses the same continuation data but requires some auxiliary data.

3.1. The computational scheme. For computational purposes a Bogdanov-Takens (BT) point is characterized by the fact that the Jacobian matrix $A=F_{u}(u, \alpha)$ has a double eigenvalue zero with geometric multiplicity one and no other eigenvalues on the imaginary axis. In other words, the characteristic polynomial $p(\lambda)=\operatorname{det}\left(A-\lambda I_{n}\right)$ satisfies

$$
\left\{\begin{array}{r}
p(0)=0 \\
p_{\lambda}(0)=0
\end{array}\right.
$$

and $A$ has rank defect 1 . Hence there exist vectors $v_{1}, w_{1} \in \mathbb{R}^{n}$ such that

$$
M(\lambda)=\left(\begin{array}{cc}
A-\lambda I_{n} & w_{1} \\
v_{1}^{T} & 0
\end{array}\right)
$$

is nonsingular in a neighborhood of $\lambda=0$. If we define $v(\lambda) \in \mathbb{R}^{n}, g(\lambda) \in$ $\mathbb{R}$ by solving

$$
M(\lambda)\left(\begin{array}{l}
v \\
g
\end{array}\right)=\left(\begin{array}{c}
0_{n} \\
1
\end{array}\right)
$$

then clearly

$$
g(\lambda)=\frac{p(\lambda)}{\operatorname{det} M(\lambda)}
$$

and by taking derivatives of (3.3) with respect to $\lambda$ we obtain

$$
\begin{gathered}
M(\lambda)\left(\begin{array}{l}
v_{\lambda} \\
g_{\lambda}
\end{array}\right)=\left(\begin{array}{c}
v \\
0
\end{array}\right), \\
M(\lambda)\left(\begin{array}{l}
v_{\lambda \lambda} \\
g_{\lambda \lambda}
\end{array}\right)=\left(\begin{array}{c}
2 v_{\lambda} \\
0
\end{array}\right) .
\end{gathered}
$$

The defining equations for the Bogdanov-Takens (BT) curve are the equilibrium equations (1.2) and

$$
\left\{\begin{array}{r}
g(0)=0 \\
g_{\lambda}(0)=0
\end{array}\right.
$$

since these are mathematically equivalent to (3.1) but are better scaled and derivatives can be computed more easily.

The continuation data are the state variables and three free parameters. The computational scheme uses $v_{1}, w_{1}$ as auxiliary data. These are initialized in the Starter and updated in the Adapter. In either case 
they are set equal to the normalized right and left singular vectors of the local Jacobian matrix $A$. In the Starter these vectors are found by an LU-decomposition of $A$, using complete pivoting. In the Adapter they are found by solving system (3.3) and a transposed system.

A BT-curve can be started from

- A BT-point (default)

- Triple zero eigenvalue

- Hopf-BT

3.2. Detection of bifurcations with higher codimension. On the BT-curve, the following singularities related to linear terms can be detected:

- Triple zero eigenvalue: $\psi_{1}=0$

- Hopf-BT: $\psi_{2}=0$

where $\psi_{i}(i=1,2)$ are defined by

$$
\left\{\begin{array}{l}
\psi_{1}=g_{\lambda \lambda} \\
\psi_{2}=g_{22}
\end{array}\right.
$$

Here $g_{\lambda \lambda}$ is obtained from (3.6). The construction of $g_{22}$ is more complicated and requires the auxiliary data $v_{1 b}, v_{2 b}, w_{1 b}, w_{2 b} \in \mathbb{R}^{m}, 2 m=$ $n(n-1)$, and scalars $d_{12}, d_{21}$ such that the matrix

$$
M_{b}=\left(\begin{array}{ccc}
2 A \odot I_{n} & w_{1 b} & w_{2 b} \\
v_{1 b}^{T} & 0 & d_{12} \\
v_{2 b}^{T} & d_{21} & 0
\end{array}\right)
$$

is nonsingular. Here $\odot$ stands for the bialternate product of two matrices; we refer to [11] and [7] for background information.

The new auxiliary data have to be initialized in the Starter and updated in the Adapter if the detection of Hopf-BT is activated. Then $g_{22}$ is computed by solving

$$
M_{b}\left(\begin{array}{c}
V \\
G
\end{array}\right)=\left(\begin{array}{c}
0_{m} \\
I_{2}
\end{array}\right)
$$

where

$$
G=\left(\begin{array}{ll}
g_{11} & g_{12} \\
g_{21} & g_{22}
\end{array}\right)
$$

4. Zero-Hopf. The chosen bifurcation data for zero-Hopf ( $\mathrm{ZH})$ points consist only of the state and parameter values; the computational scheme uses the same continuation data but requires some auxiliary data. 
4.1. The computational scheme. For computational purposes a ZH point is characterized by the fact that the Jacobian matrix $A=F_{u}(u, \alpha)$ has an algebraically simple eigenvalue zero, a pair of pure imaginary algebraically simple eigenvalues $\pm i_{0}, \omega_{0}>0$ and no other eigenvalues on the imaginary axis. This implies that there exist vectors $v_{1}, w_{1} \in \mathbb{R}^{n}$, $v_{1 b}, v_{2 b}, w_{1 b}, w_{2 b} \in \mathbb{R}^{m}, 2 m=n(n-1)$, and scalars $d_{12}, d_{21}$ such that the matrices

$$
M=\left(\begin{array}{cc}
A & w_{1} \\
v_{1}^{T} & 0
\end{array}\right)
$$

and

$$
M_{b}=\left(\begin{array}{ccc}
2 A \odot I_{n b} & w_{1 b} & w_{2 b} \\
v_{1 b}^{T} & 0 & d_{12} \\
v_{2 b}^{T} & d_{21} & 0
\end{array}\right)
$$

are nonsingular. The defining equations for the zero-Hopf curve are the equilibrium equations (1.2) and:

$$
\left\{\begin{array}{r}
g=0 \\
\operatorname{det} G=0
\end{array}\right.
$$

where the scalar $g$ results from solving

$$
M\left(\begin{array}{l}
v \\
g
\end{array}\right)=\left(\begin{array}{c}
0_{n} \\
1
\end{array}\right)
$$

and the $2 \times 2$ matrix

$$
G=\left(\begin{array}{ll}
g_{11} & g_{12} \\
g_{21} & g_{22}
\end{array}\right)
$$

is obtained by solving the system

$$
M_{b}\left(\begin{array}{l}
V \\
G
\end{array}\right)=\left(\begin{array}{c}
a_{m} \\
I_{2}
\end{array}\right)
$$

The bifurcation data are the state variables and parameters. The computational scheme uses $v_{1}, w_{1}, v_{1 b}, v_{2 b}, w_{1 b}, w_{2 b}, d_{12}, d_{21}$ as auxiliary data. These are initialized in the Starter and updated in the Adapter. The vectors $v_{1}, w_{1}$ are initialized and updated as in the BT-case. The vectors $v_{1 b}, w_{1 b}$ are chosen as normalized right and left singular vectors of $2 A \odot I_{n}$ respectively, initialized and updated essentially like $v_{1}$ and $w_{1}$. Finally, the vectors

$$
\left(\begin{array}{l}
v_{2 b} \\
d_{21}
\end{array}\right),\left(\begin{array}{l}
w_{2 b} \\
d_{12}
\end{array}\right)
$$

are updated jointly to make $M_{b}$ as well-conditioned as possible.

A zero-Hopf curve can be started from 
- A zero-Hopf point (default)

- Triple zero eigenvalue

- Hopf + BT

4.2. Detection of bifurcations with higher codimension. Along the $\mathrm{ZH}$-curve, the following test functions can be computed:

$$
\left\{\begin{array}{l}
\psi_{1}=g_{22} \\
\psi_{2}=g_{\lambda}(0)
\end{array}\right.
$$

Hereby, $g_{22}$ is defined by (4.5), (4.6) and $g_{\lambda}$ is obtained from (3.5).

The following linear singularities can be detected and located as regular zeros of the above defined test functions:

- Zero-eigenvalue + Double Hopf: $\psi_{1}=0, \psi_{2} \neq 0$

- Hopf + BT: $\psi_{1}=0, \psi_{2}=0$

- Triple zero eigenvalue: $\psi_{1} \neq 0, \psi_{2}=0$

5. Double Hopf. The chosen bifurcation data for double Hopf (DH) points consist only of the state and parameter values; the computational scheme requires some auxiliary data. For more details, see [7] and [8].

5.1. The computational scheme. For computational purposes, a double Hopf point is characterized by the fact that the Jacobian matrix $F_{u}$ has two pairs of pure imaginary algebraically simple eigenvalues $\pm i \omega_{1}, \pm i \omega_{2}, \omega_{1}, \omega_{2}>0$ and no other eigenvalues on the imaginary axis. This implies that $2 A \odot I_{n}$, where $A=F_{u}(u, \alpha)$, has rank defect 2 and there exist vectors $v_{1 b}, v_{2 b}, w_{1 b}, w_{2 b} \in \mathbb{R}^{n(n-1) / 2}$ such that the matrix

$$
M_{b}=\left(\begin{array}{ccc}
2 A \odot I_{n} & w_{1 b} & w_{2 b} \\
v_{1 b}^{T} & 0 & 0 \\
v_{2 b}^{T} & 0 & 0
\end{array}\right)
$$

is nonsingular. The defining equations for the Double Hopf curve are the equilibrium equations (1.2) and

$$
\left\{\begin{array}{l}
g_{i_{1} j_{1}}=0 \\
g_{i_{2} j_{2}}=0
\end{array}\right.
$$

with $g_{i j}=g_{i j}(u, \alpha)$ components of the matrix

$$
G=\left(\begin{array}{ll}
g_{11} & g_{12} \\
g_{21} & g_{22}
\end{array}\right)
$$

obtained by solving the system

$$
M_{b}\left(\begin{array}{c}
V \\
G
\end{array}\right)=\left(\begin{array}{c}
0_{m} \\
I_{2}
\end{array}\right) .
$$

The continuation data are the state variables and three free parameters. 
The computational scheme uses $v_{1 b}, v_{2 b}, w_{1 b}, w_{2 b}, i_{1}, j_{1}, i_{2}, j_{2}$ as auxiliary data. These are initialized in the Starter and updated in the Adapter. The vectors $v_{1 b}, v_{2 b}$ are chosen to form an orthogonal base of the right singular space of the local $2 A \odot I_{n}$ and $w_{1 b}, w_{2 b}$ similarly for the left singular space. The choice of $\left(i_{1}, j_{1}\right),\left(i_{2}, j_{2}\right)$ is such that the space spanned by the gradient vector $g_{i_{1} j_{1} z}$ has the largest component orthogonal to the equilibrium surface and $g_{i_{2} j_{2} z}$ has the largest component orthogonal to the space spanned by both the tangent space to the equilibrium surface and $g_{i_{1} j_{1} z}$; here $z$ ranges over the state variables and free parameters.

A Double Hopf curve can be started from

- A double Hopf point (default)

- Resonant double Hopf with $\omega_{1}=\omega_{2}$

- Zero-eigenvalue + double Hopf

- Hopf + BT

5.2. Detection of bifurcations with higher codimension. Along the double Hopf curve, the following test funtions can be computed:

$$
\left\{\begin{array}{l}
\psi_{1}=\operatorname{det} G^{1} \\
\psi_{2}=\operatorname{det} A \\
\psi_{3}=\langle p, q\rangle .
\end{array}\right.
$$

Hereby, the matrix $G^{1}$ is obtained by solving

$$
M_{b}\left(\begin{array}{c}
V^{1} \\
G^{1}
\end{array}\right)=\left(\begin{array}{c}
V \\
0
\end{array}\right)
$$

and $p \in \mathbb{R}^{n}$ and $q \in \mathbb{R}^{n}$ are obtained by solving

$$
\begin{gathered}
\left(\begin{array}{cc}
A & e_{p_{2}} \\
e_{p_{1}}^{T} & 0
\end{array}\right)\left(\begin{array}{c}
p \\
s
\end{array}\right)=\left(\begin{array}{c}
0_{n} \\
1
\end{array}\right) \\
\left(\begin{array}{ll}
q^{T} & s
\end{array}\right)\left(\begin{array}{cc}
A & e_{p_{2}} \\
e_{p_{1}}^{T} & 0
\end{array}\right)=\left(\begin{array}{ll}
0_{n}^{T} & 1
\end{array}\right)
\end{gathered}
$$

In (5.5) and (5.6), $e_{p_{i}}$ is the $\left(p_{i}\right)^{\text {th }}$ unit vector and $s$ denotes an irrelevant real number. The values for $p_{1}$ and $p_{2}$ are obtained by looking for the smallest pivot elements in the complete pivoting of the (Jacobian) matrix $A=F_{u}(u, \alpha)$.

The following linear singularities can be detected and located as regular zeros of the above defined test functions:

- Resonant double Hopf: $\psi_{1}=0$

- Zero-eigenvalue + double Hopf: $\psi_{2}=0, \psi_{3} \neq 0$

- Hopf + BT: $\psi_{2}=0, \psi_{3}=0$

6. Cusp. The chosen bifurcation data for cusp points consist only of the state and parameter values. The implemented computational scheme uses the same continuation data but requires some auxiliary data. 
6.1. The computational scheme. Since a cusp is a limit point where $A=F_{u}$ has rank defect 1 , there exist vectors $v_{1}, w_{1} \in \mathbb{R}^{n}$ such that

$$
M=\left(\begin{array}{cc}
A & w_{1} \\
v_{1}^{T} & 0
\end{array}\right)
$$

is nonsingular. In addition, there has to be a degeneracy in the second derivatives. The defining system of the cusp curve then consists of the equilibrium equations (1.2) together with

$$
\left\{\begin{array}{l}
g(u, \alpha)=0 \\
g^{1}(u, \alpha)=0
\end{array}\right.
$$

where $g$ is obtained by solving the single bordered $(n+1)$-dimensional system

$$
M\left(\begin{array}{l}
v(u, \alpha) \\
g(u, \alpha)
\end{array}\right)=\left(\begin{array}{c}
0_{n} \\
1
\end{array}\right)
$$

and $g^{1}$ is obtained by solving

$$
M\left(\begin{array}{c}
v^{1}(u, \alpha) \\
g^{1}(u, \alpha)
\end{array}\right)=\left(\begin{array}{c}
-B(v, v) \\
0
\end{array}\right)
$$

where the components of $B(p, q)$ are defined by

$$
B_{i}(p, q)=\left.\sum_{j, k=1}^{n} \frac{\partial^{2} F_{i}\left(u, \alpha_{0}\right)}{\partial u_{j} \partial u_{k}}\right|_{u=u_{0}} p_{j} q_{k}
$$

for $i=1,2, \ldots, n$.

The continuation data are the state variables and three free parameters. The computational scheme uses $v_{1}, w_{1}$ as auxiliary data. These are initialized in the Starter and updated in the Adapter as in section 3.

A cusp curve can be started from

- A cusp point (default)

6.2. Detection of bifurcations with higher codimension. There are no higher-codimension linear singularities detectable along the $\mathrm{CP}$ curve.

7. Generalized Hopf. The chosen bifurcation data for Generalized Hopf consist only of the state and parameter values. At present, two computational schemes are implemented to continue the GH-points.

7.1. Minimally extended system. In this method the Jacobian matrix of the defining equations is computed numerically and therefore no derivatives of order higher than three are computed symbolically; since 
the number of continuation data is small (state variables and three free parameters) and small inaccuracies in the Jacobian are usually harmless this helps to speed up the computation.

The idea behind the method is that, as in the simple Hopf case, at a generalized Hopf point the bialternate product matrix $2 A \odot I_{n}$, where $A=$ $F_{u}(u, \alpha)$, is singular. The auxiliary data are vectors $v_{1 b}, v_{2 b}, w_{1 b}, w_{2 b} \in \mathbb{R}^{n}$ and scalars $d_{12}, d_{21}$ such that the matrix

$$
M_{b}=\left(\begin{array}{ccc}
2 A \odot I_{n} & w_{1 b} & w_{2 b} \\
v_{1 b}^{T} & 0 & d_{12} \\
v_{2 b}^{T} & d_{21} & 0
\end{array}\right)
$$

is nonsingular.

The defining equations for the GH (Bautin) curve are

$$
\left\{\begin{array}{c}
F(u, \alpha)=0 \\
\operatorname{det} G=0 \\
\mathcal{L}_{0}=0
\end{array}\right.
$$

where the matrix

$$
G=\left(\begin{array}{ll}
g_{11} & g_{12} \\
g_{21} & g_{22}
\end{array}\right)
$$

is obtained by solving the system

$$
M_{b}\left(\begin{array}{c}
V \\
G
\end{array}\right)=\left(\begin{array}{c}
0_{m} \\
I_{2}
\end{array}\right)
$$

and $\mathcal{L}_{0}$ denotes the first Lyapunov coefficient, defined as

$\mathcal{L}_{0}=\operatorname{Re}\left\langle p, C(q, q, \bar{q})-2 B\left(q, A^{-1} B(q, \bar{q})\right)+B\left(\bar{q},\left(2 i \omega_{0} I_{n}-A\right)^{-1} B(q, q)\right)\right\rangle$ (see, for example [16]). The complex vectors $p, q \in \mathbb{C}^{n}$ satisfy

$A q=i \omega_{0} q, \quad A^{T} p=-i \omega_{0} p, \quad\langle\operatorname{Re} q, \operatorname{Re} q\rangle=\langle p, q\rangle=1, \quad\langle\operatorname{Re} q, \operatorname{Im} q\rangle=0$,

where this last condition is required to ensure a smooth behaviour of the vector $q$, and $\langle p, q\rangle=\bar{p}^{T} q$ is the standard scalar product in $\mathbb{d}^{n}$.

The multilinear function $B(p, q)$ is defined by $(6.5)$, while the components of $C(p, q, r)$ are defined by

$$
C_{i}(p, q, r)=\left.\sum_{j, k, l=1}^{n} \frac{\partial^{3} F_{i}\left(u, \alpha_{0}\right)}{\partial u_{j} \partial u_{k} \partial u_{l}}\right|_{u=u_{0}} p_{j} q_{k} r_{l}
$$

for $i=1,2, \ldots, n$. 
7.2. Maximally extended system. We now describe another computational scheme for Generalized Hopf that can use symbolic derivatives of order up to 4 when available. The number of continuation data is $8 n+5$ in real terms (it is convenient to express some of the equations in complex terms).

The idea behind this computational scheme is first to express explicitly that $A=F_{u}(u, \alpha)$ has an imaginary eigenvalue $\omega_{0}$ with right eigenvector $q \in \mathbb{C}^{3}$ and left eigenvector $p \in \mathbb{C}^{3}$ and then to add the condition that the first Lyapunov value vanishes. To fix the right and left eigenvectors we add the normalization conditions $\left\langle q_{0}, q\right\rangle=\langle p, q\rangle=1$, where $q_{0} \in \mathbb{C}^{n}$ is the normalized right eigenvector $q$ at a previously computed point on the curve. To simplify formally the expression for $\mathcal{L}_{0}$, we introduce $v \in \mathbb{R}^{n}, w \in \mathbb{C}^{n}$ as additional unknowns, where

$$
v=A^{-1} B(q, \bar{q}), \quad w=\left(2 i w_{0} I_{n}-A\right)^{-1} B(q, q)
$$

Thus, the continuation data for this computational scheme consist of the state and parameter variables and the components of

$$
\left(q, p, v, w, \omega_{0}, \lambda\right)
$$

The components of the vector $q_{0}$ are considered as auxiliary data.

The defining equations for the Generalized-Hopf (Bautin) curve then are given by (1.2) and the complex system:

$$
\left\{\begin{aligned}
A q-i \omega_{0} q & =0 \\
A^{T} p+\lambda p & =0 \\
\langle q 0, q\rangle-1 & =0 \\
\langle p, q\rangle-1 & =0 \\
A v-B(q, \bar{q}) & =0 \\
\left(2 \omega_{0} I_{n}-A\right) w-B(q, q) & =0 \\
\operatorname{Re}\langle p, C(q, q, \bar{q})-2 B(q, v)+B(\bar{q}, w)\rangle & =0
\end{aligned}\right.
$$

The multilinear functions $B(p, q)$ and $C(p, q, r)$ are defined by $(6.5)$ and (7.5). The complex variable $\lambda$ is introduced artificially to regularize the system; formally, along the GH curve, $\lambda=i \omega_{0}$. When written in the real form, (1.2) and (7.6) together form a system of $8 n+5$ equations with $8 n+6$ variables (including three free parameters).

A Generalized Hopf curve can be started from

- A Generalized Hopf point (default)

7.3. Detection of bifurcations with higher codimension. There are no higher-codimension linear singularities detectable along the GHcurve. 
8. Discussion. We described the continuation of generic codimension-2 equilibrium bifurcations of (1.1) with detection of the codimension-3 equilibrium bifurcations due to degenerations in the linear terms of (1.1).

In addition, figure 1 gives some other equilibrium bifurcations that can be computed in CONTENT (see also the examples). In particular, branching points can be detected and computed on equilibrium curves. Generically, these are codimension-2 points but their appearance in examples (due to symmetry-breaking phenomena) is so common that they are included.

Explicit normal form coefficients for all codim 2 equilibrium bifurcations are derived in [17]. They provide test functions to detect the remaining codim 3 bifurcations of equilibria. Three of these are already implemented in the present release of CONTENT, namely the swallowtail (ST), generically found on cusp curves and the double (ZB) and triple (ZA) equilibrium degenerate Bogdanov-Takens bifurcations. Both are generically found on curves of BT-points and ZA is also found on cusp curves.

\section{Examples.}

9.1. LP newron model. The LP neuron is the Lateral Pyloric neuron of the stomatogastric ganglion of the crab Cancer Borealis. A complete description of the model (with 13 state variables and 29 parameters), developed at Cornell University, is given in [7].

Starting from the point with coordinates given in table 1, CONTENT draws a curve of Bogdanov-Takens points as shown in figure 2, where HBT denotes a Hopf + Bogdanov-Takens point found on the curve. While following the curve, the parameters $I_{e x t}, \bar{g}_{A f}$ and $\bar{g}_{K(C a)}$ are free. [7].

The parameters not given in table 1 are fixed at their values given in

TABLE 1

Coordinates of starting point for Bogdanov-Takens curve.

\begin{tabular}{|c|c|c|c|c|}
\hline & state & value & param & value \\
\hline 1 & $v$ & -49.02293 & Iext & 3.398637 \\
2 & $h$ & 0.6646423 & $\bar{g}_{A f}$ & 12.96179 \\
3 & $C a$ & 0.1179416 & $\bar{g}_{K(C a)}$ & 5 \\
4 & $a_{C a_{1}}$ & 0.4355952 & & \\
5 & $a_{C a_{2}}$ & $3.922597 \mathrm{e}-05$ & & \\
6 & $b_{C a_{1}}$ & 0.4695044 & & \\
7 & $n$ & 0.1957435 & & \\
8 & $a_{K(C a)}$ & 0.0001300977 & & \\
9 & $b_{K(C a)}$ & 0.8357226 & & \\
10 & $a_{A}$ & 0.4069875 & & \\
11 & $b_{A f}$ & 0.1031369 & & \\
12 & $b_{A s}$ & 0.1031369 & & \\
13 & $a_{h}$ & 0.006172727 & & \\
\hline
\end{tabular}


CONTINUATION OF CODIMENSION-2 EQUILIBRTUM BIFURCATIONS 175

Starting from the point with coordinates given in table 2, we find a curve of Zero-Hopf points as shown in figure 3, where HBT denotes a Hopf + Bogdanov-Takens point found on the curve.

TABLE 2

Coordinates of starting point for Zero-Hopf curve.

\begin{tabular}{|c|c|c|c|c|}
\hline & state & value & param & value \\
\hline 1 & $v$ & -37.2233 & Iext & 1.014954 \\
2 & $h$ & 0.09159829 & $\bar{g}_{A f}$ & 3.675916 \\
3 & $C a$ & 0.1719147 & $\bar{g}_{K(C a)}$ & 5 \\
4 & $a_{C a_{1}}$ & 0.02306315 & & \\
5 & $a_{C a_{2}}$ & 0.0002116275 & & \\
6 & $b_{C a_{1}}$ & 0.1683891 & & \\
7 & $n$ & 0.3276093 & & \\
8 & $a_{K(C a)}$ & 0.001798594 & & \\
9 & $b_{K(C a)}$ & 0.777288 & & \\
10 & $a_{A}$ & 0.5892931 & & \\
11 & $b_{A f}$ & 0.01583688 & & \\
12 & $b_{A s}$ & 0.01583688 & & \\
13 & $a_{h}$ & 0.002318011 & & \\
\hline
\end{tabular}

TABLE 3

Coordinates of starting point for cusp curve in LP neuron model.

\begin{tabular}{|c|c|c|c|c|}
\hline & state & value & param & value \\
\hline 1 & $v$ & -35.56432 & Iext & 1.098998 \\
2 & $h$ & 0.06847848 & $\bar{g}_{A f}$ & 4.097115 \\
3 & $C a$ & 0.1779745 & $\bar{g}_{K(C a)}$ & 5.260545 \\
4 & $a_{C a_{1}}$ & 0.02905193 & & \\
5 & $a_{C a_{2}}$ & 0.0002682095 & & \\
6 & $b_{C a_{1}}$ & 0.141309 & & \\
7 & $n$ & 0.3494562 & & \\
8 & $a_{K(C a)}$ & 0.002503221 & & \\
9 & $b_{K(C a)}$ & 0.7712336 & & \\
10 & $a_{A}$ & 0.6141357 & & \\
11 & $b_{A f}$ & 0.01205739 & & \\
12 & $b_{A s}$ & 0.01205739 & & \\
13 & $a_{h}$ & 0.002019319 & & \\
\hline
\end{tabular}

Starting from the cusp point with coordinates given in table 3, we can compute a curve of cusp points as shown in figure 4 . Hereby, the parameters $I_{e x t}, \bar{g}_{A f}$ and $\bar{g}_{K(\mathrm{Ca})}$ were kept free. 
In figure 5, a three dimensional projection of the same cusp curve is shown.

9.2. Bykov-Yablonskii-Kim model. The second example is a model of oxidation of $\mathrm{CO}$ on $\mathrm{Pt}$ [3] given by the following equations

$$
\left\{\begin{array}{l}
\dot{x}=2 q_{1} z^{2}-2 q_{5} x^{2}-q_{3} x y \\
\dot{y}=q_{2} z-q_{6} y-q_{3} x y \\
\dot{s}=q_{4} z-k q_{4} s
\end{array}\right.
$$

where $z=1-x-y-s$. Starting from a Bogdanov-Takens point with coordinates given in table 4 , CONTENT computed a BT curve on which a triple equilibrium ( $\mathrm{ZA}$ ) and double equilibrium ( $\mathrm{ZB}$ ) degenerate BogdanovTakens points were found. Starting from a generalized Hopf point with coordinates given in table 5 , we computed a curve of $\mathrm{GH}$ points. Both curves are shown in figure 6.

\section{TABLE 4}

Coordinates of starting point for Bogdonou-Takens curve in Bykov model.

\begin{tabular}{|c|c|c|c|c|}
\hline & state & value & param & value \\
\hline 1 & $x$ & 0.01633704 & $q_{1}$ & 2.5 \\
2 & $y$ & 0.6384092 & $q_{2}$ & 1.161198 \\
3 & $s$ & 0.2004567 & $q_{3}$ & 10 \\
4 & & & $q_{4}$ & 0.0675 \\
5 & & & $q_{5}$ & 1 \\
6 & & & $q_{6}$ & 0.1 \\
7 & & & $k$ & 0.7223359 \\
\hline
\end{tabular}

TABLe 5

Coordinates of starting point for generalized Hopf curve in Bykov model.

\begin{tabular}{|c|c|c|c|c|}
\hline & state & value & param & value \\
\hline 1 & $x$ & 0.03335851 & $q_{1}$ & 0.9674027 \\
2 & $y$ & 0.3639242 & $q_{2}$ & 0.6242381 \\
3 & $s$ & 0.3499418 & $q_{3}$ & 10 \\
4 & & & $q_{4}$ & 0.0675 \\
5 & & & $q_{5}$ & 1 \\
6 & & & $q_{6}$ & 0.1 \\
7 & & & $k$ & 0.7223359 \\
\hline
\end{tabular}

9.3. A codim 3 normal form. This model (see [2], [5]) has two state variables $(x, y)$ and three parameters $(a, b, c)$. It is defined by

$$
\left\{\begin{array}{l}
\dot{x}=y \\
\dot{y}=a+b x+c y-x^{3}+x y-x^{2} y
\end{array}\right.
$$


Hereby, the user defined function $a^{2}+b^{2}+c^{2}-r$ is attached to this system. Starting with a Hopf point with coordinates given in table 6 , we calculated a curve of Hopf points, with parameters $a, b, c$ free and $r=0.16$ fixed, on which one GH and two BT points were detected. Following a curve of $\mathrm{BT}$ points from one of these BT points with $r$ freed, we got a BT curve on which a triple equilibrium point (ZA) was detected. Starting a curve of limit points, we found a closed curve containing two cusp (CP) points as well as the two earlier found Bogdanov-Takens points. All three curves are shown in figure 7.

TABLE 6

Coordinates of starting point for Hopf curve in codim 9 mode!.

\begin{tabular}{|c|c|c|c|c|}
\hline & state & value & param & value \\
\hline 1 & $x$ & 0.4505103 & $a$ & -0.04842174 \\
2 & $y$ & 0 & $b$ & 0.3104415 \\
3 & & & $c$ & -0.2475508 \\
4 & & & $r$ & 0.16 \\
\hline
\end{tabular}

9.4. New Lorenz model. The New Lorenz model of atmospheric circulation $[18]$ has 3 state variables $(x, y, z)$ and 4 parameters $(a, b, F, G)$ and is defined by

$$
\left\{\begin{array}{l}
\dot{x}=-y^{2}-z^{2}-a x+a F \\
\dot{y}=x y-b x z-y+G \\
\dot{z}=b x y+x z-z
\end{array}\right.
$$

Starting from a zero-Hopf point with coordinates given in table 7 and with $b, F$ and $G$ free, we followed a zero Hopf curve on which a point with triple zero eigenvalue (TZ) was found as well as a curve of Hopf points containing two zero-Hopf points. Both curves are shown in figure 8 .

TABLE 7

Coordinates of starting point for Hopf and zero-Hopf curves in New Lorenz model.

\begin{tabular}{|c|c|c|c|c|}
\hline & state & value & param & value \\
\hline 1 & $x$ & 1.125 & $a$ & 0.25 \\
2 & $y$ & -0.01038068 & $b$ & 4 \\
3 & $z$ & 0.3737046 & $F$ & 1.684052 \\
4 & & & $G$ & 1.682968 \\
\hline
\end{tabular}

9.5. Steinmetz-Larter model. The Steinmetz-Larter model de scribing the peroxidase-oxidase reaction [22] has 4 state variables $(a, b, x, y)$ and 9 parameters $\left(k_{1}, k_{2}, k_{3}, k_{4}, k_{5}, k_{6}, k_{7}, k_{8}, k_{9}\right)$ and is defined by 


$$
\left\{\begin{array}{l}
\dot{a}=-k_{1} a b x-k_{3} a b y+k_{7}-k_{9} a \\
\dot{b}=-k_{1} a b x-k_{3} a b y+k_{8} \\
\dot{x}=k_{1} a b x-2 k_{2} x^{2}+2 k_{3} a b y-k_{4} x+k_{6} \\
\dot{y}=-k_{3} a b y+2 k_{2} x^{2}-k_{5} y
\end{array}\right.
$$

Starting from a generalized Hopf point with coordinates given in table 8, CONTENT was able to compute a curve of generalized Hopf points as shown in figure 9 . For continuation, the parameters $k_{6}, k_{7}, k_{8}$ were free.

TABLE 8

Coordinates of starting point for generalized Hopf curve in Steinmetz-Larter model.

\begin{tabular}{|c|c|c|c|c|}
\hline & state & value & param & value \\
\hline 1 & $a$ & 50.40856 & $k_{1}$ & 0.1631021 \\
2 & $b$ & 0.9790055 & $k_{2}$ & 1250 \\
3 & $x$ & 0.01342439 & $k_{3}$ & 0.046875 \\
4 & $y$ & 0.1318399 & $k_{4}$ & 20 \\
5 & & & $k_{5}$ & 1.104 \\
6 & & & $k_{6}$ & 0.001 \\
7 & & & $k_{7}$ & 6.336045 \\
8 & & & $k_{8}$ & 0.4130391 \\
9 & & & $k_{9}$ & 0.1175 \\
\hline
\end{tabular}


CONTINUATION OF CODIMENSION-2 EQUILIBRIUM BIFURCATIONS 179

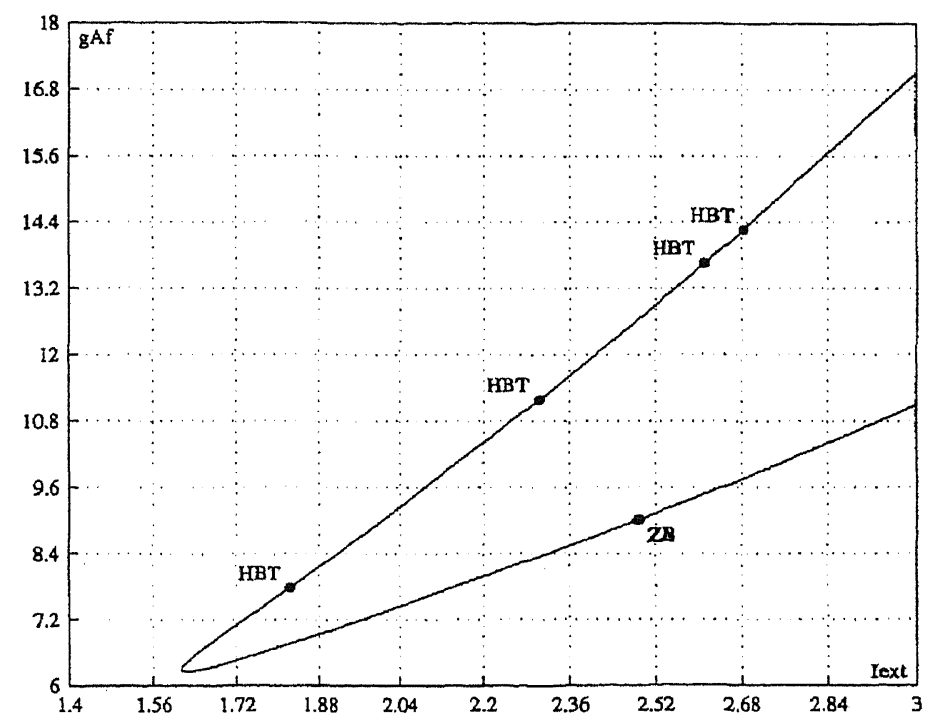

FIG. 2. BT curve in LP neuron model.

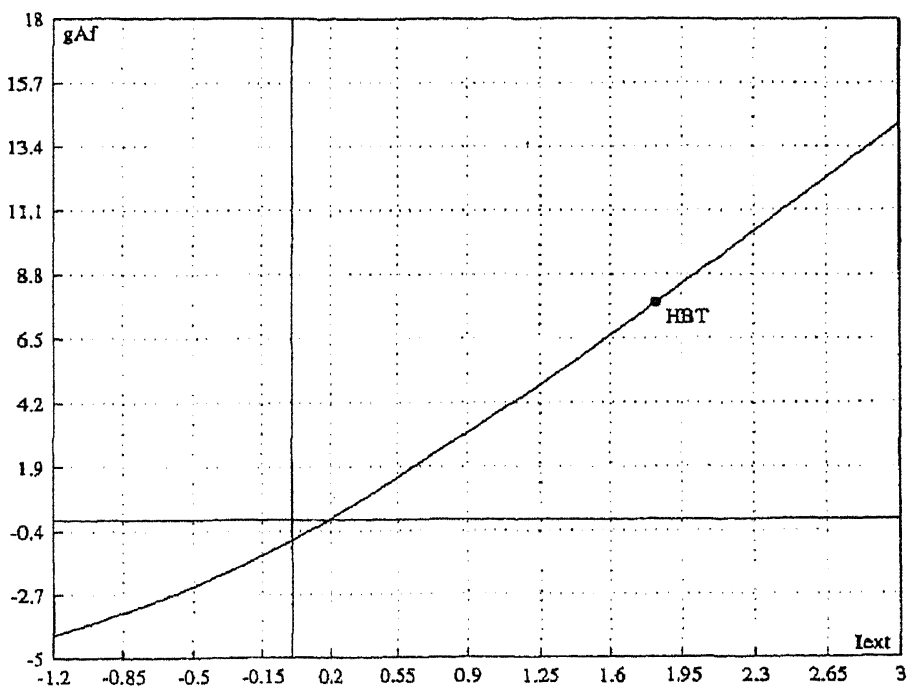

FIG. 3. Zero-Hopf curve in LP neuron model. 


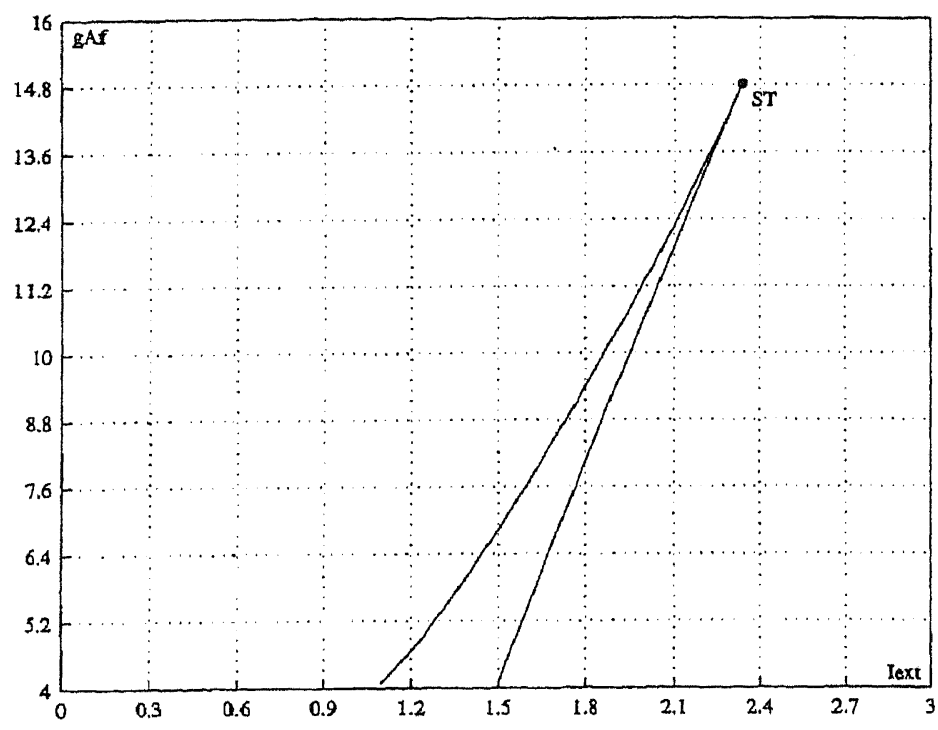

F1G. 4. Cusp curve in LP neuron model.

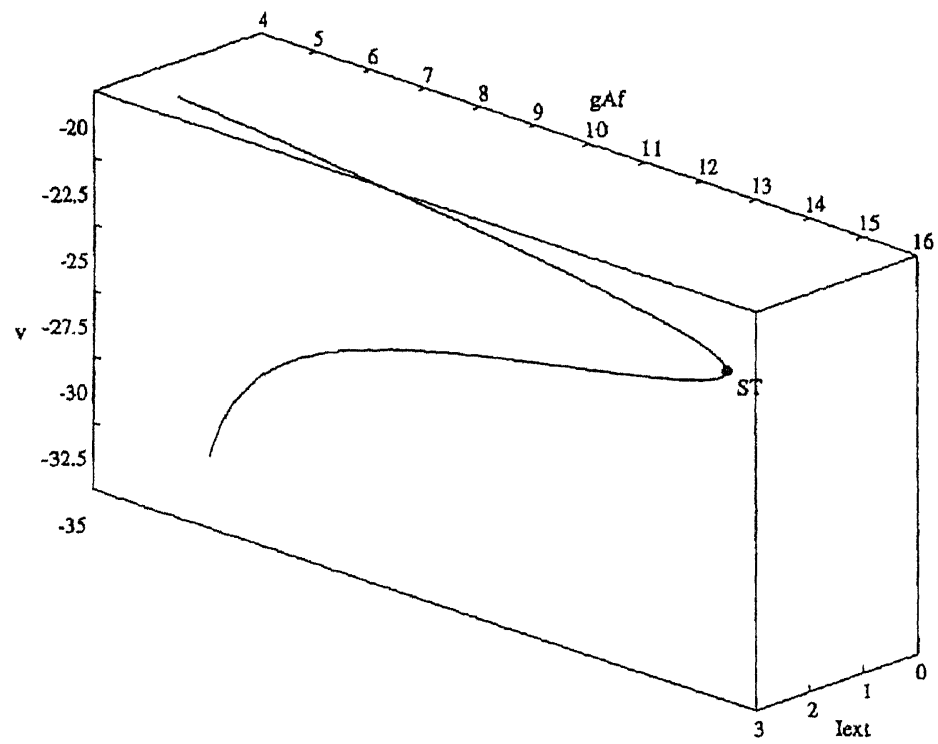

FiG. 5. Cusp curve in $3 D$ for LP neuron model. 


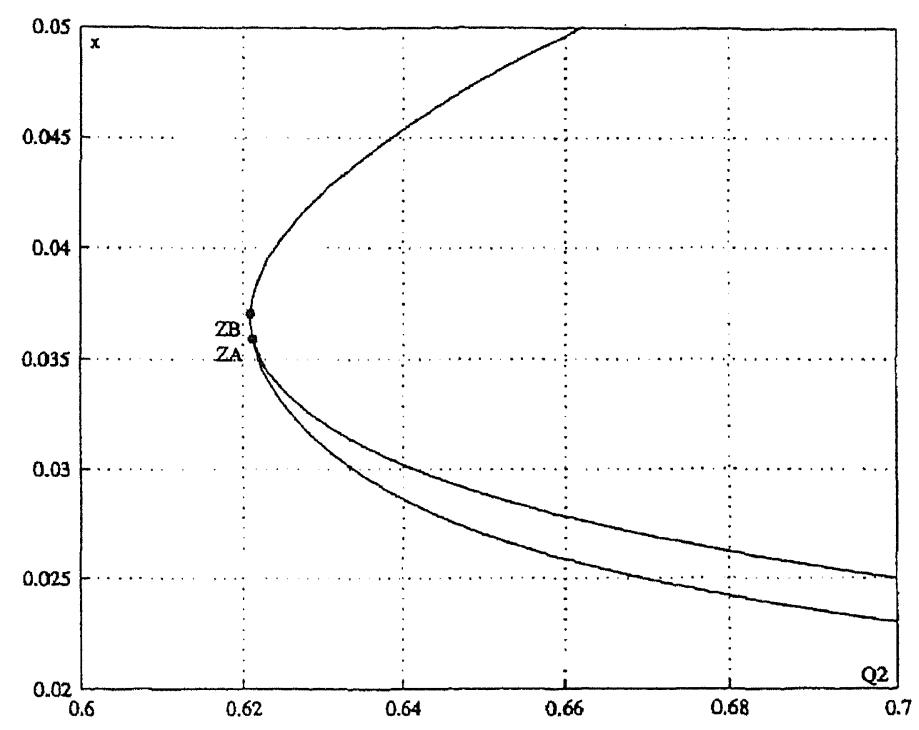

FrG. 6. Bogdanov-Takens and Generalized Hopf curve in Bykov model.

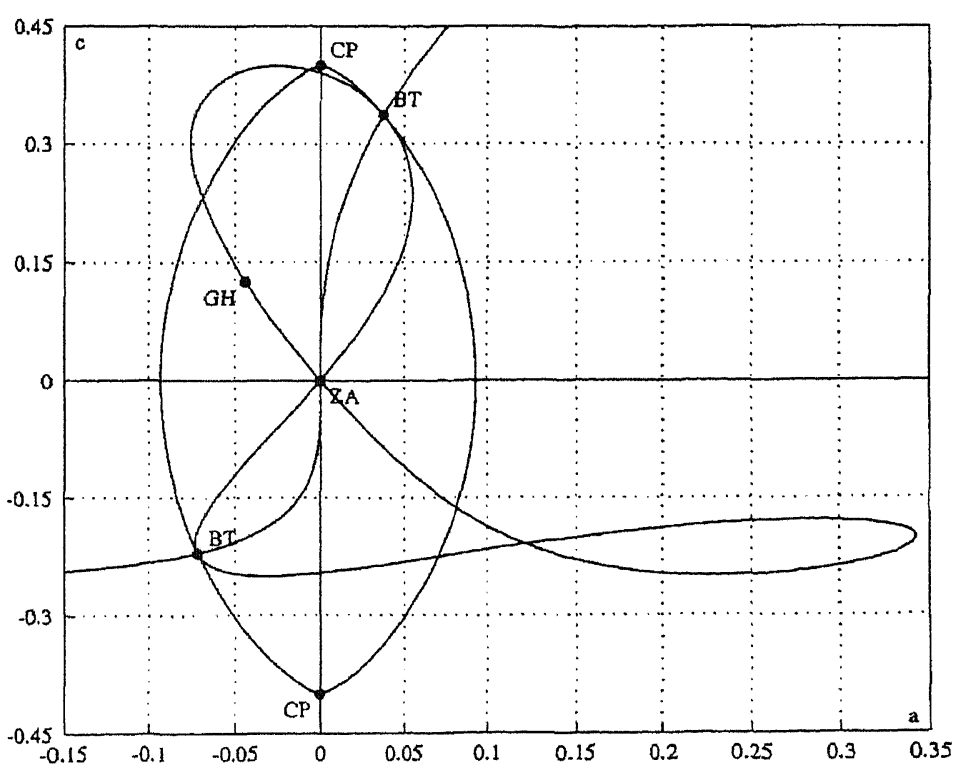

FIG. 7. Limit point, Hopf and Bogdanov-Takens curves in codim 3 model. 


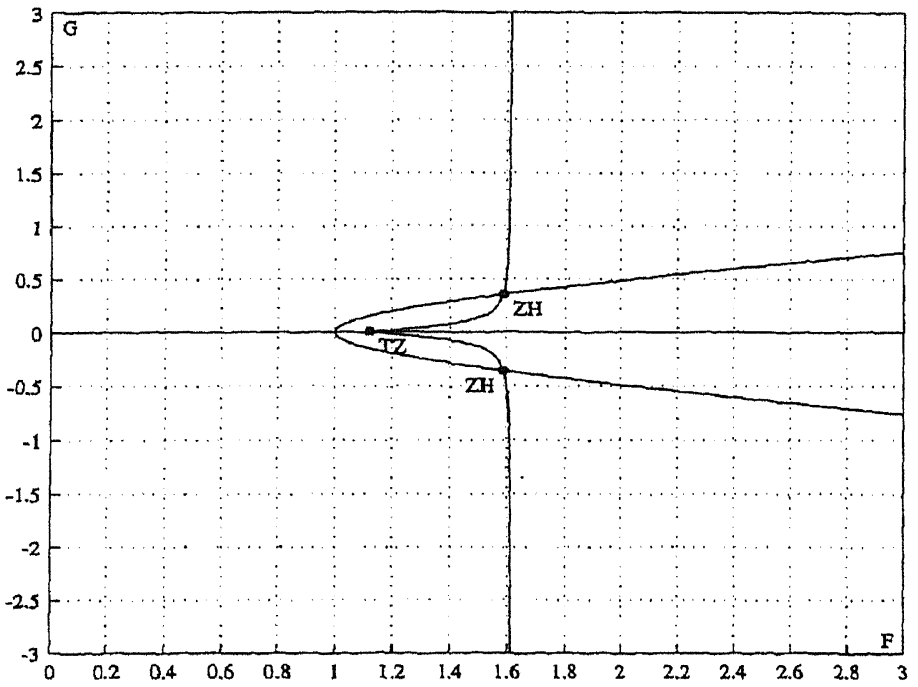

FyG. 8. Hopf and Zero-Hopf curves in New Lorenz model.

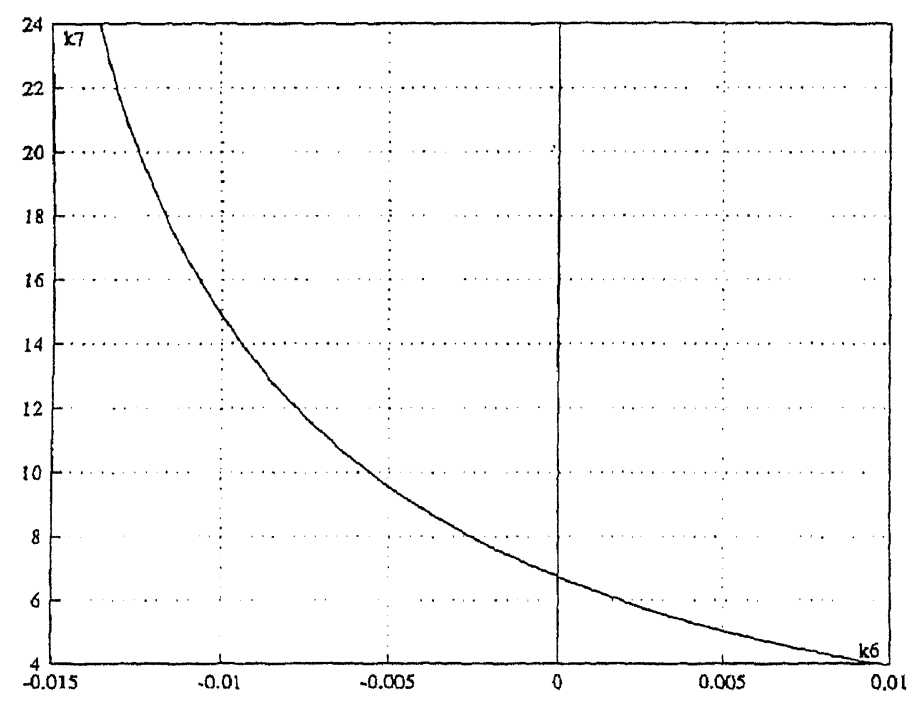

FrG. 9. Generalized Hopf curve in Steinmetz-Larter model. 
[1] W. J. BEYN, Numerical Methods for dynamical systems, Advances in Numerical Analysis, Vol I: Nonlinear partial differential equations and dynamical systems, (ed. W. Light), Clarendon Press, Oxford, (1991), 175-236.

[2] A. D. BazyKIn, YU. A. Kuznetsov, AND A. I. KhIBnik, Portraits of Bifutcations: Bifurcation Diagrams of Planar Dynamical Systems, Znanie, Moscow, (1989), [in Russian].

[3] V. I. BYKov, G. S. YABLONSKII, AND V. F. KIM, On the simple model of kinetic self-oscillations in catalitic reaction of $C O$ oxidation, Dokl. AN SSSR, 242, (1978), [in Russian], 637-639.

[4] K. CHU, W. GovaERTS AND A. SPENCE, Matrices with rank deficiency two in eigenvalue problems and dynamical systems, SIAM J. Numer. Anal., 31, (1994), $524-539$.

[5] F. Dumortier, R. ROUSSARIE, J. SOTOMAYOR, AND H. ŻOLADEK, Bifurcations of Planar Vector Fields, Nilpotent Singularities and Abelian Integrals, Lect. Notes in Math., 1480, Springer-Verlag, Berlin, Heidelberg, (1991).

[6] W. GovaERTs, Computation of Takens-Bogdanov type bifurcations with arbitrary codimension, SIAM J. Numer. Anal., 30, (1993), 1121-1133.

[7] W. Govaerts, J. GuCKenHeImer, AND A. KHIBNIK, Defining functions for multiple Hopf bifurcations, SIAM J. Numer. Anal., 34, (1997), 1269-1288.

[8] W. GOVAERTS, YU. A. KUzNeTsoV, AND B. SiJnave, Implementation of Hopf and double Hopf continuation using bordering methods, to appear in ACM Transactions on Mathematical Software.

[9] A. Griewank aNd G. W. Reddien, Computation of cusp singularities for operator equations and their discretizations, J. Comput. Appl. Maths., 26, (1989), 133153.

[10] J. GuCKenhemer and P. Holmes, Nonlinear Oscillations, Dynamical Systems, and Bifurcations of Vector Fields, Applied Mathematical Sciences, 42, Springer Verlag, (1983).

[11] J. GuCKenfeImer, M. MYers aND B. Sturmfels, Computing Hopf Bifurcations I, SIAM J. Numer. Anal., 34, (1997), 1-21.

[12] M. HOLODNIOK AND M. KUBIČEK, New algorithms for the evaluation of complex bifurcation points in ordinary differential equations. A comparative numerical study, Appl. Math. Comput., 15, (1984), 261-274.

[13] A. JEPSON AND A. SPENCE, Singular poirts and their computation, Numerical methods for bifurcation problems (eds. $\mathbf{T}$. Küpper, $H$. Mittelmann and $H$. Weber) Birkhäuser ISNM, 70, (1984), 195-209.

[14] H. B. KELLER, The bordering algorithm and path following near singular points of higher nullity, SIAM J. Sci. Stat. Comp., 4, (1983), 573-582.

[15] M. KUBIC̈EK AND M. HOLODNIOK, Numerical determination of bifurcation points in steady state and periodic solutions-numerical algorithms and examples, Numerical methods for bifurcation problems (eds. T. Küpper, H. Mittelmann and H. Weber) Birkhäuser ISNM, 70, (1984), 247-270.

[16] Yu. A. KuzNeTSOV, Elements of Applied Bifurcation Theory, Applied Mathematical Sciences, 112, Springer Verlag, (1995).

[17] YU. A. KuzNeTsov, Explicit Normal Form Coefficients for all Codim 2 Bifurcations of Equilibrio in ODEs, CWI Report MAS-9730, (http://www.cwi.nl/static/publications/reports/abs/MAS-R9730.html).

[18] E.Lonenz, Irregularity: a fundamental property of the atmosphere, Tellus $36 \mathrm{~A}$ (1984), 98-110.

[19] G. MOORE AND A. SPENCE, The calculation of turning points of nonlinear equations, SLAM J. Numer. Anal., 17, (1980), 567-576.

[20] D. RoOsE AND V. HLAVAČEK, $A$ direct method for the computation of Hopf bifurcation points, SIAM J. Appl. Math., 45, (1985), 879-894.

[21] R. SEYDEL, Numerical computation of branch points in nonlinear equations, $\mathrm{Nu}$ 
mer. Math., 33, (1979), 339-352

[22] C. G. STEINMBT2, AND R. LARTER, The quasiperiodic route to chaos in a model of the peroxidase-oxidase reaction, J. Chem. Phys., 94, (1991), 1388-1396.

[23] B. WERNER, Computation of Hopf bifurcation with bordered matrices, SIAM J. Numer. Anal., 33, (1996), 435-455. 